\title{
Crypt Glandular Lumen
}

National Cancer Institute

\section{Source}

National Cancer Institute. Crypt Glandular Lumen. NCI Thesaurus. Code C83195.

The lumen of the glandular structures of a crypt. 